\title{
Competition-Enhanced Ligand Selection to Screen for DNA Aptamers for Spherical Gold
} Nanoparticles

\section{SUPPORTING INFORMATION}

Maeling Tapp ${ }^{1}$, Patrick Dennis ${ }^{2}$, Rajesh R. Naik ${ }^{3}$, and Valeria T. Milam ${ }^{1,4, *}$

\author{
${ }^{1}$ School of Materials Science and Engineering \\ ${ }^{4}$ Petit Institute for Bioengineering and Bioscience
}

Georgia Institute of Technology, 771 Ferst Dr. NW, Atlanta, GA 30332-0245, USA

${ }^{2}$ Materials \& Manufacturing Directorate, Soft Matter Materials Branch, Air Force Research Laboratory

Wright-Patterson AFB, OH 45433, USA

${ }^{3} 711^{\text {th }}$ Human Performance Wing, Air Force Research Laboratory

Wright-Patterson AFB, OH 45433, USA

*Corresponding Author: Email: valeria.milam@mse.gatech.edu 


\section{Gold nanoparticle characterization}

Spherical gold nanoparticles (AuNPs) were analyzed via UV-vis spectroscopy at 11 days postaging from gold nanoparticle seeds. Samples were also prepared for TEM analysis (JEOL $100 \mathrm{CX}$ II $100 \mathrm{kV}$ ) in order to estimate average diameter using ImageJ software (http:// rsbweb.nih.gov/ij/) by measuring the diameter of ca. 150 particles.
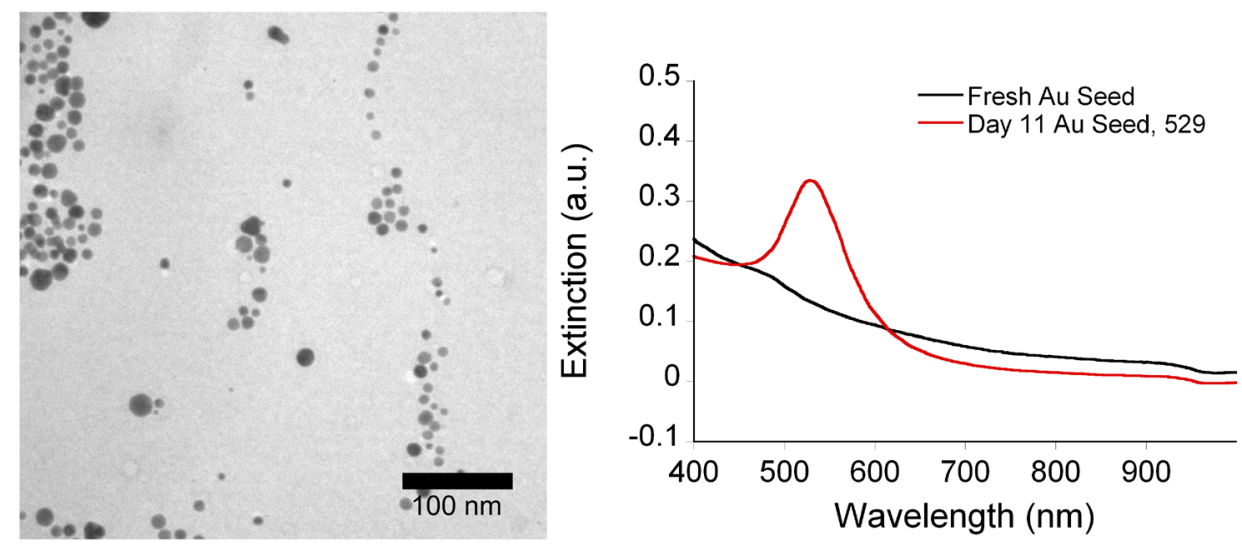

Figure S1. TEM micrograph of gold seed aged for 11 days (AuNPs) with accompanying UVVis spectra with resulting peak wavelength value included in the legend. 


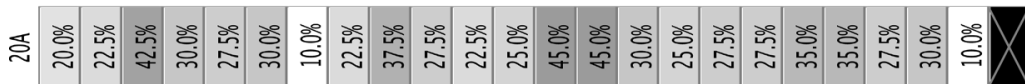

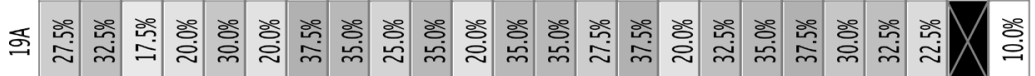

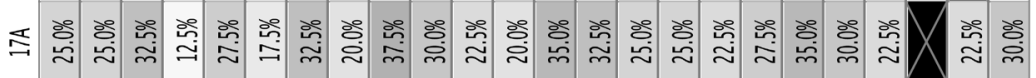

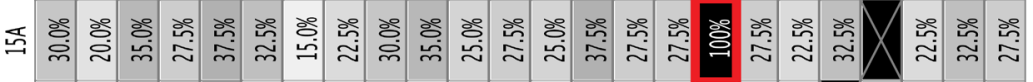

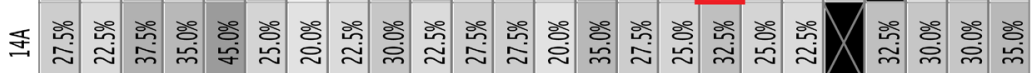

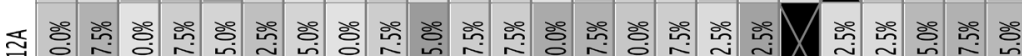
m m

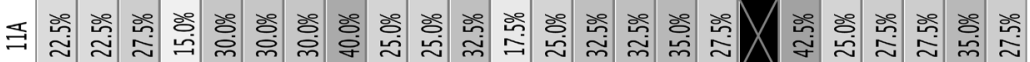

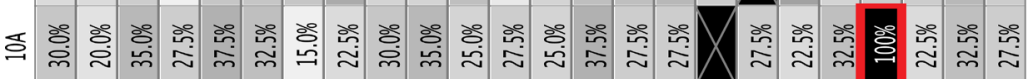

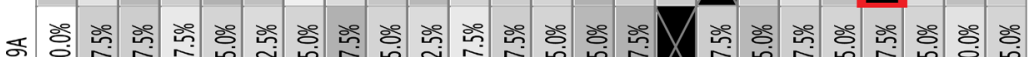
๙

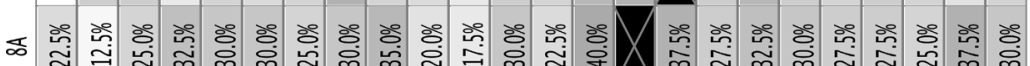

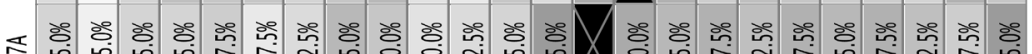

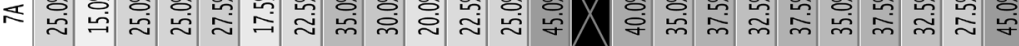

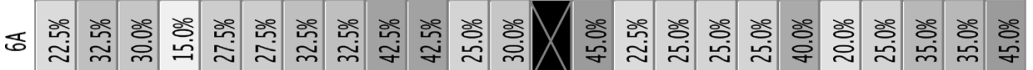

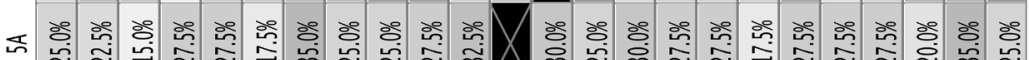

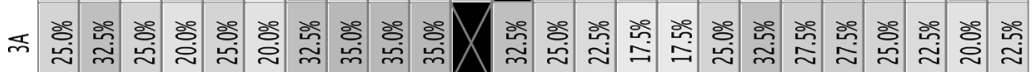

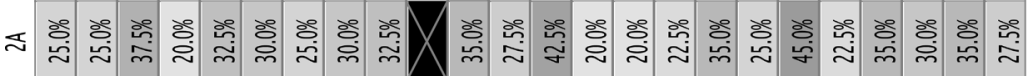

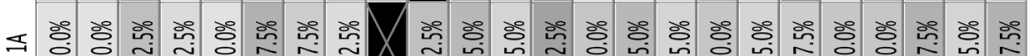

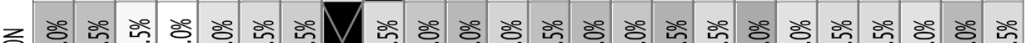

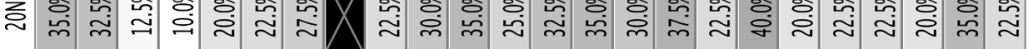

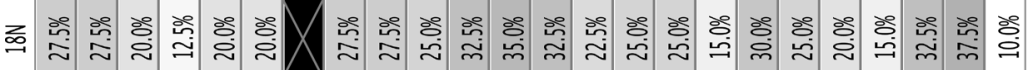

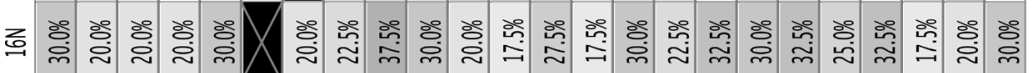

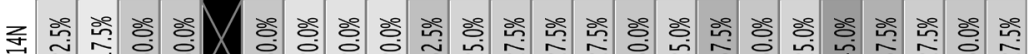

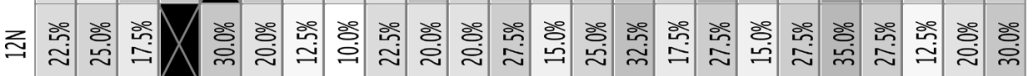

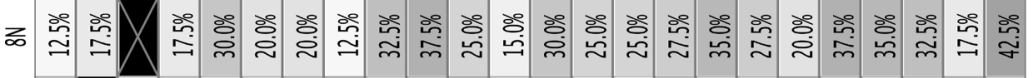

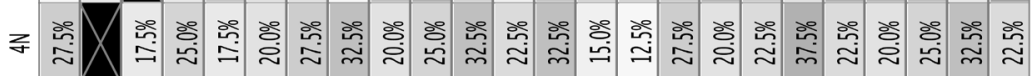

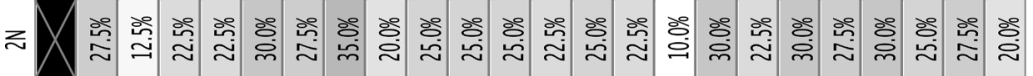

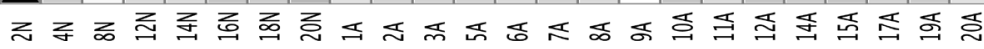

Figure S2. Primary sequence pairwise distance matrix for all aptamer candidates. Generated with Geneious 7.1.2 created by Biomatters. Available from http://www.geneious.com. 
Aptamer candidates selected from the normal library

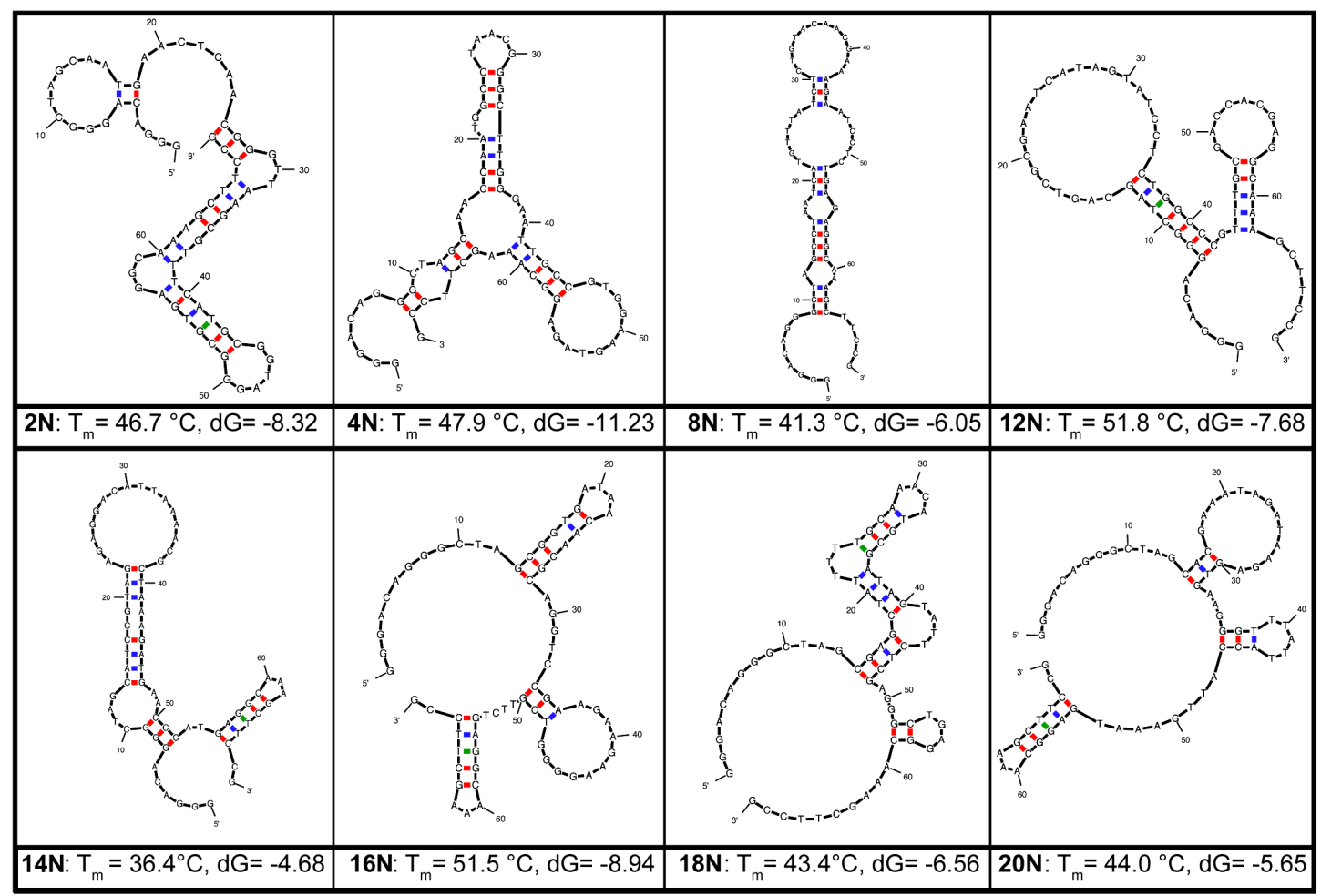

Figure S3. Thermodynamically favorable secondary structures of the 8 aptamer sequences selected from the normal random library ( $25 \%$ of each base). Predicted structures were generated using UNAFOLD (http://mfold.rna.albany.edu/?q=mfold/DNA-Folding-Form, access date: July $23,2014)$ under the following solution conditions: $\mathrm{T}=23{ }^{\circ} \mathrm{C}, 152 \mathrm{mM}\left[\mathrm{Na}^{+}\right], 4 \mathrm{mM}\left[\mathrm{Mg}^{++}\right]$. The position-specific (with base 1 at the $5^{\prime}$ end and base 69 at the $3^{\prime}$ end) base pairings are colorcoded as follows: A-T and T-A (blue); G-C and C-G (red); G-T and T-G (green). The Gibbs free energy associated with self-hybridization, $\mathrm{dG}$, is provided in units of $\mathrm{kcal} / \mathrm{mol}$. 
Aptamer candidates selected from the A-rich library

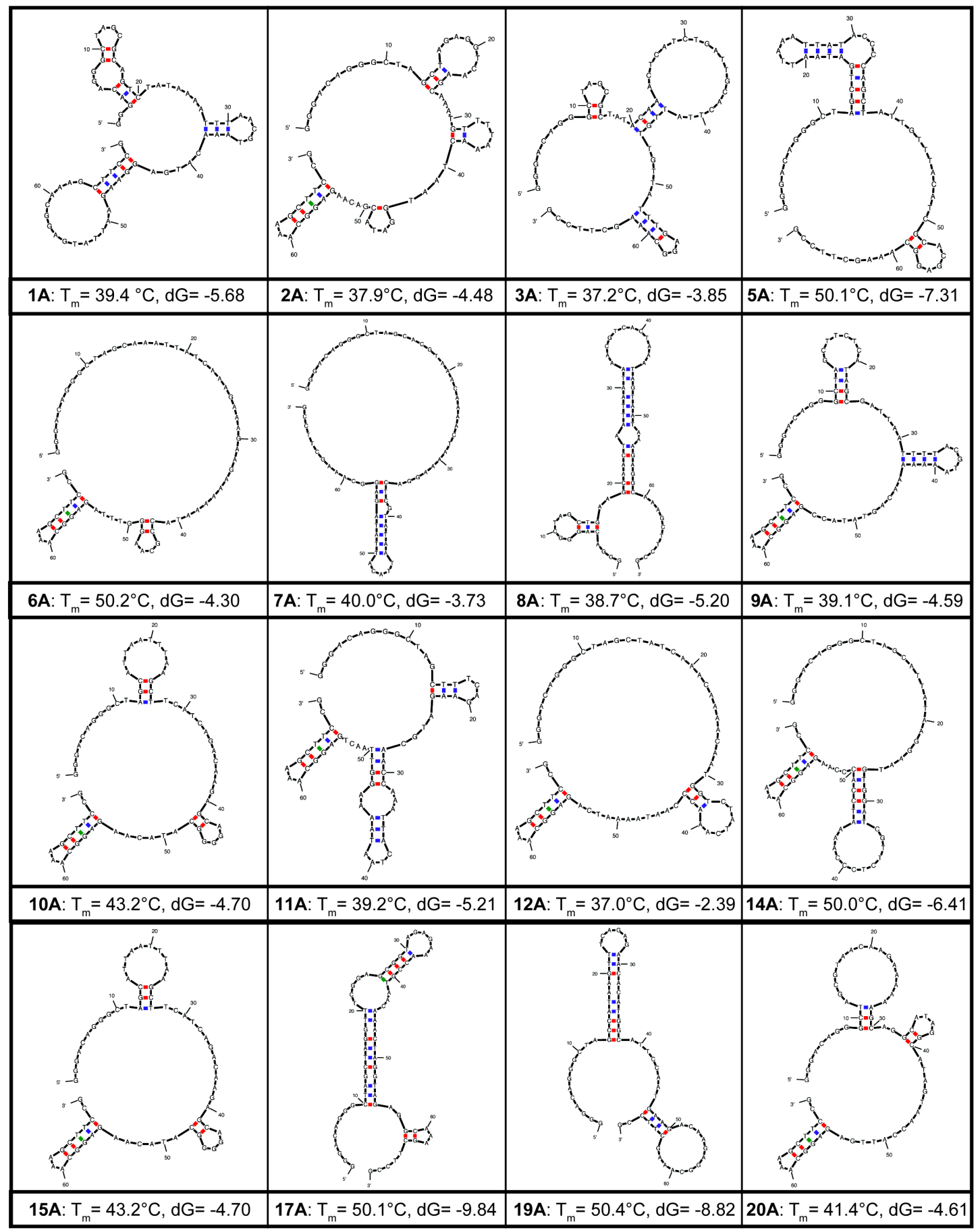

Figure S4. Thermodynamically favorable secondary structures of 16 aptamer sequences selected from the A-rich random library (40\% A, 20\% each of C, T, G). Predicted structures were generated using UNAFOLD (http://mfold.rna.albany.edu/?q=mfold/DNA-Folding-Form, access date: July 23 , 2014) under the following solution conditions: $\mathrm{T}=23{ }^{\circ} \mathrm{C}, 152 \mathrm{mM}\left[\mathrm{Na}^{+}\right], 4 \mathrm{mM}$ $\left[\mathrm{Mg}^{++}\right]$. The position-specific (with base 1 at the $5^{\prime}$ end and base 69 at the $3^{\prime}$ end) base pairings are color-coded as follows: A-T and T-A (blue); G-C and C-G (red); G-T and T-G (green). The Gibbs free energy associated with self-hybridization, $\mathrm{dG}$, is provided in units of $\mathrm{kcal} / \mathrm{mol}$. 


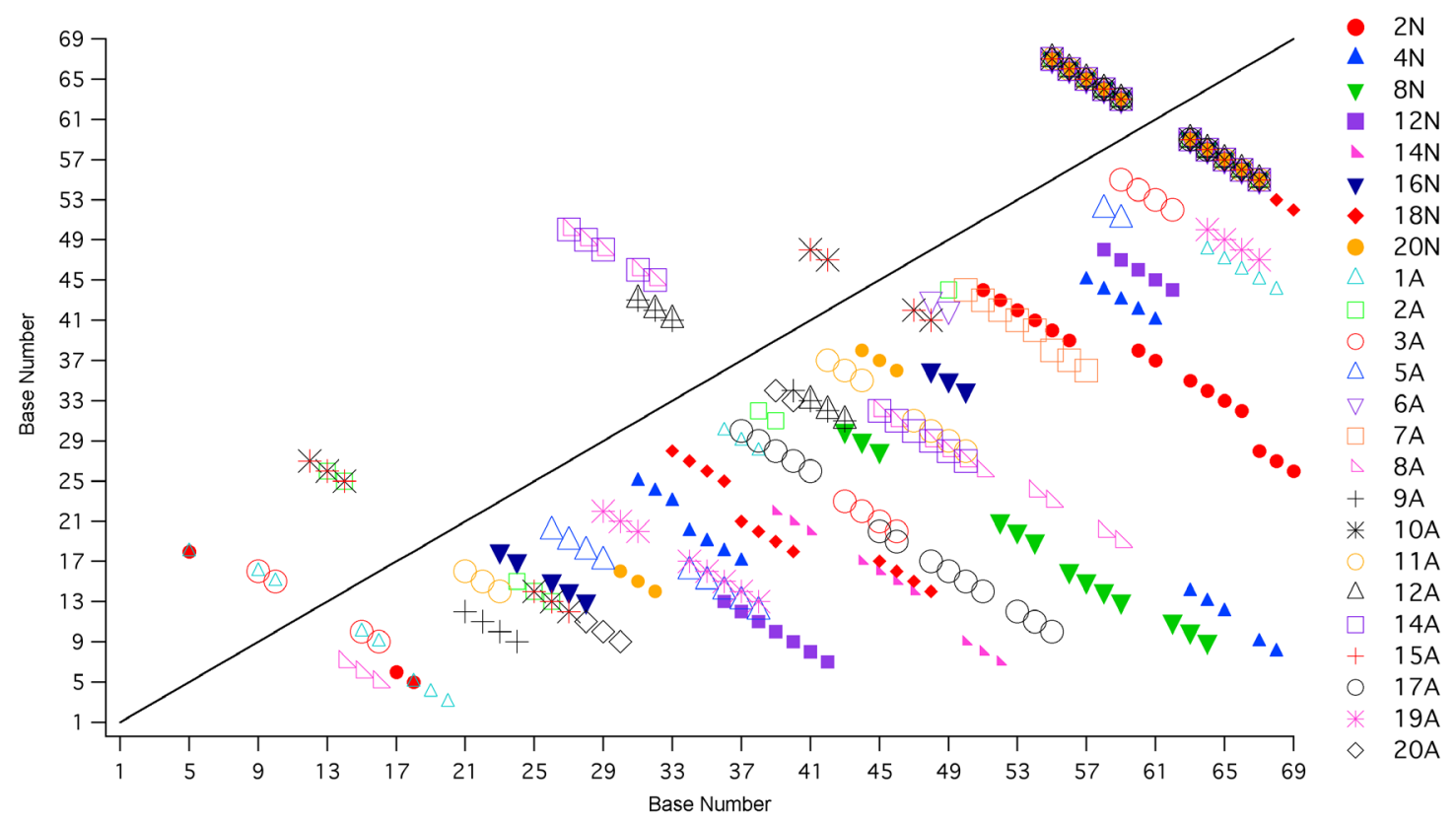

Figure S5. Base-pair map highlighting all base pair interactions for each aptamer candidate (below the line) and the shared base pair interactions between aptamer candidates (above the line). Detailed lists of all shared and unshared position-dependent base pair interactions for all 24 sequences are provided in Supporting Information Tables S1 \& S2.

Table S1. List of the position-dependent identities of base pairs shared by two or more aptamer sequences. Numerical base positions in red indicate positions within central variable segment of the sequence.

\begin{tabular}{|c|c|c|c|c|c|c|c|c|c|c|c|c|c|}
\hline Base Pair & \begin{tabular}{|l|} 
Sequence \\
(Pair Type)
\end{tabular} & $\begin{array}{l}\text { Sequence } \\
\text { (Pair Type) }\end{array}$ & \begin{tabular}{l|} 
Sequence \\
(Pair Type)
\end{tabular} & $\begin{array}{l}\text { Sequence } \\
\text { (Pair Type) }\end{array}$ & $\begin{array}{l}\text { Sequence } \\
\text { (Pair Type) }\end{array}$ & \begin{tabular}{|l|} 
Sequence \\
(Pair Type)
\end{tabular} & \begin{tabular}{|l|} 
Sequence \\
(Pair Type)
\end{tabular} & $\begin{array}{l}\text { Sequence } \\
\text { (Pair Type) }\end{array}$ & $\begin{array}{l}\text { Sequence } \\
\text { (Pair Type) }\end{array}$ & $\begin{array}{l}\text { Sequence } \\
\text { (Pair Type) }\end{array}$ & $\begin{array}{l}\text { Sequence } \\
\text { (Pair Type) }\end{array}$ & $\begin{array}{l}\text { Sequence } \\
\text { (Pair Type) }\end{array}$ & $\begin{array}{l}\text { Sequence } \\
\text { (Pair Type) }\end{array}$ \\
\hline 5,18 & $2 \mathrm{~N}(\mathrm{C}-\mathrm{G})$ & $1 A(C-G)$ & & & & & & & & & & & \\
\hline 9,16 & $1 \mathrm{~A}(\mathrm{G}-\mathrm{C})$ & $3 A(G-C)$ & & & & & & & & & & & \\
\hline 10,15 & $1 \mathrm{~A}(\mathrm{C}-\mathrm{G})$ & $3 A(C-G)$ & & & & & & & & & & & \\
\hline 12,27 & $10 \mathrm{~A}(\mathrm{~A}-\mathrm{T})$ & $15 \mathrm{~A}(\mathrm{~A}-\mathrm{T})$ & & & & & & & & & & & \\
\hline 13,26 & $2 A(G-C)$ & $10 \mathrm{~A}(\mathrm{G}-\mathrm{C})$ & $15 A(G-C)$ & & & & & & & & & & \\
\hline 14,25 & $2 A(C-G)$ & $10 A(C-G)$ & $15 \mathrm{~A}(\mathrm{C}-\mathrm{G})$ & & & & & & & & & & \\
\hline 27,50 & $8 \mathrm{~A}(\mathrm{~T}-\mathrm{A})$ & $14 \mathrm{~A}(\mathrm{G}-\mathrm{C})$ & & & & & & & & & & & \\
\hline 28,49 & $8 \mathrm{~A}(\mathrm{~T}-\mathrm{A})$ & $14 \mathrm{~A}(\mathrm{~T}-\mathrm{A})$ & & & & & & & & & & & \\
\hline 29,48 & $8 \mathrm{~A}(\mathrm{~A}-\mathrm{T})$ & $14 \mathrm{~A}(\mathrm{G}-\mathrm{C})$ & & & & & & & & & & & \\
\hline 31,43 & $9 A(T-A)$ & $12 \mathrm{~A}(\mathrm{G}-\mathrm{C})$ & & & & & & & & & & & \\
\hline 31,46 & $8 \mathrm{~A}(\mathrm{~T}-\mathrm{A})$ & $14 \mathrm{~A}(\mathrm{~A}-\mathrm{T})$ & & & & & & & & & & & \\
\hline 32,42 & $9 A(T-A)$ & $12 \mathrm{~A}(\mathrm{G}-\mathrm{C})$ & & & & & & & & & & & \\
\hline 32,45 & $8 A(A-T)$ & $14 \mathrm{~A}(\mathrm{~T}-\mathrm{A})$ & & & & & & & & & & & \\
\hline 33,41 & $9 \mathrm{~A}(\mathrm{~T}-\mathrm{A})$ & $12 \mathrm{~A}(\mathrm{~T}-\mathrm{A})$ & & & & & & & & & & & \\
\hline 41,48 & $10 \mathrm{~A}(\mathrm{G}-\mathrm{C})$ & $15 A(G-C)$ & & & & & & & & & & & \\
\hline 42.47 & $10 \mathrm{~A}(\mathrm{C}-\mathrm{G})$ & $15 A(C-G)$ & & & & & & & & & & & \\
\hline 55,67 & $14 \mathrm{~N}(\mathrm{G}-\mathrm{C})$ & $16 \mathrm{~N}(\mathrm{G}-\mathrm{C})$ & $20 \mathrm{~N}(\mathrm{G}-\mathrm{C})$ & $2 A(G-C)$ & $6 \mathrm{~A}(\mathrm{G}-\mathrm{C})$ & $9 A(G-C)$ & $10 A(G-C)$ & $11 \mathrm{~A}(\mathrm{G}-\mathrm{C})$ & $12 \mathrm{~A}(\mathrm{G}-\mathrm{C})$ & $14 \mathrm{~A}(\mathrm{G}-\mathrm{C})$ & $15 \mathrm{~A}(\mathrm{G}-\mathrm{C})$ & $20 \mathrm{~A}(\mathrm{G}-\mathrm{C})$ & \\
\hline 56,66 & $14 \mathrm{~N}(\mathrm{C}-\mathrm{G})$ & $16 \mathrm{~N}(\mathrm{C}-\mathrm{G})$ & $20 \mathrm{~N}(\mathrm{C}-\mathrm{G})$ & $2 A(C-G)$ & $6 A(C-G)$ & $9 A(C-G)$ & $10 A(C-G)$ & $11 \mathrm{~A}(\mathrm{C}-\mathrm{G})$ & $12 A(C-G)$ & $14 \mathrm{~A}(\mathrm{C}-\mathrm{G})$ & $15 \mathrm{~A}(\mathrm{C}-\mathrm{G})$ & $20 \mathrm{~A}(\mathrm{C}-\mathrm{G})$ & \\
\hline 57,65 & $14 \mathrm{~N}(\mathrm{~T}-\mathrm{G})$ & $16 \mathrm{~N}(\mathrm{~T}-\mathrm{G})$ & $20 \mathrm{~N}(\mathrm{~T}-\mathrm{G})$ & $2 A(T-G)$ & $6 \mathrm{~A}(\mathrm{~T}-\mathrm{G})$ & $9 \mathrm{~A}(\mathrm{~T}-\mathrm{G})$ & $10 \mathrm{~A}(\mathrm{~T}-\mathrm{G})$ & $11 \mathrm{~A}(\mathrm{~T}-\mathrm{G})$ & $12 \mathrm{~A}(\mathrm{~T}-\mathrm{G})$ & $14 \mathrm{~A}(\mathrm{~T}-\mathrm{G})$ & $15 A(T-G)$ & $20 \mathrm{~A}(\mathrm{~T}-\mathrm{G})$ & \\
\hline 58,64 & $14 \mathrm{~N}(\mathrm{~T}-\mathrm{A})$ & $16 \mathrm{~N}(\mathrm{~T}-\mathrm{A})$ & $20 \mathrm{~N}(\mathrm{~T}-\mathrm{A})$ & $2 \mathrm{~A}(\mathrm{~T}-\mathrm{A})$ & $6 \mathrm{~A}(\mathrm{~T}-\mathrm{A})$ & $9 A(T-A)$ & $10 \mathrm{~A}(\mathrm{~T}-\mathrm{A})$ & $11 \mathrm{~A}(\mathrm{~T}-\mathrm{A})$ & $12 \mathrm{~A}(\mathrm{~T}-\mathrm{A})$ & $14 \mathrm{~A}(\mathrm{~T}-\mathrm{A})$ & $15 \mathrm{~A}(\mathrm{~T}-\mathrm{A})$ & $17 \mathrm{~A}(\mathrm{~T}-\mathrm{A})$ & $20 \mathrm{~A}(\mathrm{~T}-\mathrm{A})$ \\
\hline 59,63 & $14 \mathrm{~N}(\mathrm{C}-\mathrm{G})$ & $16 \mathrm{~N}(\mathrm{C}-\mathrm{G})$ & $20 \mathrm{~N}(\mathrm{C}-\mathrm{G})$ & $2 \mathrm{~A}(\mathrm{C}-\mathrm{G})$ & $6 A(C-G)$ & $9 A(C-G)$ & $10 \mathrm{~A}(\mathrm{C}-\mathrm{G})$ & $11 \mathrm{~A}(\mathrm{C}-\mathrm{G})$ & $12 \mathrm{~A}(\mathrm{C}-\mathrm{G})$ & $14 \mathrm{~A}(\mathrm{C}-\mathrm{G})$ & $15 \mathrm{~A}(\mathrm{C}-\mathrm{G})$ & $17 \mathrm{~A}(\mathrm{C}-\mathrm{G})$ & $20 \mathrm{~A}(\mathrm{C}-\mathrm{G})$ \\
\hline
\end{tabular}


Table S2. List of all aptamer sequences and the total number of position-dependent base pairs shared with other aptamer sequences as well as the total number and nomenclature of aptamer sequences with at least one shared base pairing for a given aptamer sequence.

\begin{tabular}{|c|c|c|}
\hline Sequence & $\begin{array}{c}\text { Total number } \\
\text { of shared base } \\
\text { pair positions }\end{array}$ & $\begin{array}{l}\text { Number (and aptamer nomenclature) of } \\
\text { sequences with at least one shared base pair }\end{array}$ \\
\hline $2 \mathrm{~N}$ & 1 & $1(1 \mathrm{~A}$ \\
\hline $4 \mathrm{~N}$ & 0 & 0 \\
\hline $8 \mathrm{~N}$ & 0 & 0 \\
\hline $12 \mathrm{~N}$ & 0 & 0 \\
\hline $14 \mathrm{~N}$ & 5 & $12(16 \mathrm{~N}, 20 \mathrm{~N}, 2 \mathrm{~A}, 6 \mathrm{~A}, 9 \mathrm{~A}, 10 \mathrm{~A}, 11 \mathrm{~A}, 12 \mathrm{~A}, 14 \mathrm{~A}, 15 \mathrm{~A}, 17 \mathrm{~A}, 20 \mathrm{~A}$ \\
\hline $16 \mathrm{~N}$ & 5 & $12(14 \mathrm{~N}, 20 \mathrm{~N}, 2 \mathrm{~A}, 6 \mathrm{~A}, 9 \mathrm{~A}, 10 \mathrm{~A}, 11 \mathrm{~A}, 12 \mathrm{~A}, 14 \mathrm{~A}, 15 \mathrm{~A}, 17 \mathrm{~A}, 20 \mathrm{~A}$ \\
\hline $18 \mathrm{~N}$ & 0 & 0 \\
\hline $20 \mathrm{~N}$ & 5 & $12(14 \mathrm{~N}, 16 \mathrm{~N}, 2 \mathrm{~A}, 6 \mathrm{~A}, 9 \mathrm{~A}, 10 \mathrm{~A}, 11 \mathrm{~A}, 12 \mathrm{~A}, 14 \mathrm{~A}, 15 \mathrm{~A}, 17 \mathrm{~A}, 20 \mathrm{~A}$ \\
\hline $1 \mathrm{~A}$ & 3 & $2(2 \mathrm{~N}, 3 \mathrm{~A})$ \\
\hline 2A & 7 & $12(14 \mathrm{~N}, 16 \mathrm{~N}, 20 \mathrm{~N}, 6 \mathrm{~A}, 9 \mathrm{~A}, 10 \mathrm{~A}, 11 \mathrm{~A}, 12 \mathrm{~A}, 14 \mathrm{~A}, 15 \mathrm{~A}, 17 \mathrm{~A}, 20 \mathrm{~A}$ \\
\hline $3 \mathrm{~A}$ & 2 & $1(1 \mathrm{~A}$ \\
\hline 5A & 0 & 0 \\
\hline 6A & 5 & $12(14 \mathrm{~N}, 16 \mathrm{~N}, 20 \mathrm{~N}, 2 \mathrm{~A}, 9 \mathrm{~A}, 10 \mathrm{~A}, 11 \mathrm{~A}, 12 \mathrm{~A}, 14 \mathrm{~A}, 15 \mathrm{~A}, 17 \mathrm{~A}, 20 \mathrm{~A}$ \\
\hline 7A & 0 & 0 \\
\hline 8A & 5 & $1(14 \mathrm{~A}$ \\
\hline 9A & 8 & $12(14 \mathrm{~N}, 16 \mathrm{~N}, 20 \mathrm{~N}, 2 \mathrm{~A}, 6 \mathrm{~A}, 10 \mathrm{~A}, 11 \mathrm{~A}, 12 \mathrm{~A}, 14 \mathrm{~A}, 15 \mathrm{~A}, 17 \mathrm{~A}, 20 \mathrm{~A}$ \\
\hline $10 \mathrm{~A}$ & 10 & $12(14 \mathrm{~N}, 16 \mathrm{~N}, 20 \mathrm{~N}, 2 \mathrm{~A}, 6 \mathrm{~A}, 9 \mathrm{~A}, 11 \mathrm{~A}, 12 \mathrm{~A}, 14 \mathrm{~A}, 15 \mathrm{~A}, 17 \mathrm{~A}, 20 \mathrm{~A}$ \\
\hline $11 \mathrm{~A}$ & 5 & $12(14 \mathrm{~N}, 16 \mathrm{~N}, 20 \mathrm{~N}, 2 \mathrm{~A}, 6 \mathrm{~A}, 9 \mathrm{~A}, 10 \mathrm{~A}, 12 \mathrm{~A}, 14 \mathrm{~A}, 15 \mathrm{~A}, 17 \mathrm{~A}, 20 \mathrm{~A}$ \\
\hline 12A & 8 & $12(14 \mathrm{~N}, 16 \mathrm{~N}, 20 \mathrm{~N}, 2 \mathrm{~A}, 6 \mathrm{~A}, 9 \mathrm{~A}, 10 \mathrm{~A}, 11 \mathrm{~A}, 14 \mathrm{~A}, 15 \mathrm{~A}, 17 \mathrm{~A}, 20 \mathrm{~A}$ \\
\hline 14A & & $\mathrm{N}, 16 \mathrm{~N}, 20 \mathrm{~N}, 2 \mathrm{~A}, 6 \mathrm{~A}, 8 \mathrm{~A}, 9 \mathrm{~A}, 10 \mathrm{~A}, 11 \mathrm{~A}, 12 \mathrm{~A}, 14 \mathrm{~A}, 15 \mathrm{~A}, 17 \mathrm{~A}, 20 \mathrm{~A}$ \\
\hline 15A & 10 & $12(14 \mathrm{~N}, 16 \mathrm{~N}, 20 \mathrm{~N}, 2 \mathrm{~A}, 6 \mathrm{~A}, 9 \mathrm{~A}, 10 \mathrm{~A}, 11 \mathrm{~A}, 12 \mathrm{~A}, 14 \mathrm{~A}, 17 \mathrm{~A}, 20 \mathrm{~A}$ \\
\hline $17 \mathrm{~A}$ & 2 & $12(14 \mathrm{~N}, 16 \mathrm{~N}, 20 \mathrm{~N}, 2 \mathrm{~A}, 6 \mathrm{~A}, 9 \mathrm{~A}, 10 \mathrm{~A}, 11 \mathrm{~A}, 12 \mathrm{~A}, 14 \mathrm{~A}, 15 \mathrm{~A}, 20 \mathrm{~A}$ \\
\hline 19A & 0 & 0 \\
\hline 20A & 5 & $12(14 \mathrm{~N}, 16 \mathrm{~N}, 20 \mathrm{~N}, 2 \mathrm{~A}, 6 \mathrm{~A}, 9 \mathrm{~A}, 10 \mathrm{~A}, 11 \mathrm{~A}, 12 \mathrm{~A}, 14 \mathrm{~A}, 15 \mathrm{~A}, 17 \mathrm{~A}$ \\
\hline
\end{tabular}


Table S3. List of each aptamer sequence and the total number of hairpins (H), interior loops (IL), bulges (B), duplexes (D) and single-stranded segments (SS) found in its predicted secondary structure. The total number of the 5 structural features observed for each sequence (right) as well as across all sequences (bottom) is provided.

\begin{tabular}{|c|c|c|c|c|c|c|c|c|c|c|c|c|c|c|c|c|c|c|}
\hline & \multicolumn{17}{|c|}{ Secondary Structure Features } & \\
\hline Sequence & $1 \mathrm{H}$ & $2 \mathrm{H}$ & $3 \mathbf{H}$ & $4 \mathrm{H}$ & 1IL & 2IL & 3IL & 1B & 2B & 1D & 2D & 3D & $2 S S$ & $35 s$ & 4SS & $5 \mathrm{SS}$ & $1 \mathrm{MBL}$ & Total \\
\hline $2 \mathrm{~N}$ & & $\mathrm{x}$ & & & $\mathrm{x}$ & & & & $\mathrm{x}$ & & & $\mathrm{x}$ & $\mathrm{X}$ & & & & & 5 \\
\hline $4 \mathrm{~N}$ & & $\mathrm{x}$ & & & $\mathrm{x}$ & & & $\mathrm{x}$ & & & & $x$ & $x$ & & & & $\mathrm{x}$ & 6 \\
\hline $8 \mathbf{N}$ & $\mathrm{x}$ & & & & & & $\mathrm{x}$ & & & & & $\mathrm{x}$ & $\mathrm{x}$ & & & & & 4 \\
\hline $12 \mathrm{~N}$ & & $x$ & & & & & & & & & & & & $\mathrm{x}$ & & & & 2 \\
\hline $14 \mathrm{~N}$ & & $\mathrm{X}$ & & & & $\mathrm{x}$ & & & & & $\mathrm{x}$ & & & $\mathrm{X}$ & & & & 4 \\
\hline $16 \mathrm{~N}$ & & & $\mathrm{X}$ & & $\mathrm{x}$ & & & & & $\mathrm{x}$ & & & & & $\mathrm{x}$ & & & 4 \\
\hline $18 \mathrm{~N}$ & & $\mathrm{X}$ & & & & & & & $\mathrm{X}$ & & $\mathrm{X}$ & & & $\mathrm{X}$ & & & & 4 \\
\hline $20 \mathrm{~N}$ & & & $\mathrm{x}$ & & & & & & & & & & & & $\mathrm{x}$ & & & 2 \\
\hline 1A & & & $\mathrm{X}$ & & $\mathrm{X}$ & & & & & $\mathrm{X}$ & & & & & $\mathrm{x}$ & & & 4 \\
\hline $2 \mathrm{~A}$ & & & & $\mathrm{x}$ & & & & & & & & & & & & $\mathrm{x}$ & & 2 \\
\hline $3 \mathrm{~A}$ & & & $\mathrm{X}$ & & & & & & & & & & & & $\mathrm{X}$ & & & 2 \\
\hline $5 A$ & & $\mathrm{x}$ & & & & & & $\mathrm{x}$ & & $\mathrm{x}$ & & & & $\mathrm{x}$ & & & & 4 \\
\hline $6 \mathrm{~A}$ & & $\mathrm{x}$ & & & & & & & & & & & & $\mathrm{X}$ & & & & 2 \\
\hline $7 \mathrm{~A}$ & $\mathrm{x}$ & & & & & & & $x$ & & $X$ & & & $X$ & & & & & 4 \\
\hline $8 \mathrm{~A}$ & & $X$ & & & & & $X$ & & & & & $X$ & & $X$ & & & & 4 \\
\hline $9 \mathrm{~A}$ & & & $x$ & & & & & & & & & & & & $x$ & & & 2 \\
\hline $10 \mathrm{~A}$ & & & $x$ & & & & & & & & & & & & $X$ & & & 2 \\
\hline $11 \mathrm{~A}$ & & & $X$ & & $X$ & & & & & $X$ & & & & & $X$ & & & 4 \\
\hline $12 \mathrm{~A}$ & & $X$ & & & & & & & & & & & & $x$ & & & & 2 \\
\hline $14 \mathrm{~A}$ & & $X$ & & & & & & & & & & & & $X$ & & & & 2 \\
\hline $15 \mathrm{~A}$ & & & $x$ & & & & & & & & & & & & $x$ & & & 2 \\
\hline $17 \mathrm{~A}$ & & $x$ & & & & & $x$ & & & & & $x$ & & $x$ & & & & 4 \\
\hline $19 \mathrm{~A}$ & & $x$ & & & $X$ & & & & & $x$ & & & & $x$ & & & & 4 \\
\hline $20 \mathrm{~A}$ & & & $x$ & & & & & & & & & & & & $x$ & & & 2 \\
\hline Total & 2 & 12 & 9 & 1 & 6 & 1 & 3 & 3 & 2 & 6 & 2 & 5 & 4 & 10 & 9 & 1 & 1 & \\
\hline$\%$ & 8.33 & 50.00 & 37.50 & 4.17 & 25.00 & 4.17 & 12.50 & 12.50 & 8.33 & 25.00 & 8.33 & 20.83 & 16.67 & 41.67 & 37.50 & 4.17 & 4.17 & \\
\hline
\end{tabular}

